\title{
Distinguishing epigenetic features of preneoplastic testis tissues adjacent to seminomas and nonseminomas
}

\author{
Ildar V. Gainetdinov ${ }^{1, *}$, Sofia A. Kondratieva ${ }^{1, *}$, Yulia V. Skvortsova ${ }^{1}$, Marina V. \\ Zinovyeva $^{1}$, Elena A. Stukacheva ${ }^{1}$, Alexey Klimov², Alexey A. Tryakin ${ }^{3}$, Tatyana L. \\ Azhikina ${ }^{1}$ \\ ${ }^{1}$ Department of Genetics and Postgenomic Technologies, Shemyakin-Ovchinnikov Institute of Bioorganic Chemistry, Russian \\ Academy of Sciences, Moscow, Russia \\ ${ }^{2}$ Department of Oncology, Blokhin Russian Cancer Research Center, Moscow, Russia \\ ${ }^{3}$ Department of Clinical Pharmacology and Chemotherapy, Blokhin Russian Cancer Research Center, Moscow, Russia \\ *These authors have contributed equally to this work \\ Correspondence to: Ildar V. Gainetdinov, e-mail: ildargv@gmail.com
}

Keywords: PIWI, testicular germ cell tumor, DNA methylation, LINE-1, DDX4

Received: September 24, $2015 \quad$ Accepted: January 12, 2016

Published: January 29, 2016

ABSTRACT

PIWI pathway proteins are expressed during spermatogenesis where they play a key role in germ cell development. Epigenetic loss of PIWI proteins expression was previously demonstrated in testicular germ cell tumors (TGCTs), implying their involvement in TGCT development. In this work, apart from studying only normal testis and TGCT samples, we also analyzed an intermediate stage, i.e. preneoplastic testis tissues adjacent to TGCTs. Importantly, in this study, we minimized the contribution of patient-to-patient heterogeneity by using matched preneoplastic/TGCT samples. Surprisingly, expression of germ cell marker DDX4 suggests that spermatogenesis is retained in premalignant testis tissues adjacent to nonseminoma, but not those adjacent to seminoma. Moreover, this pattern is followed by expression of PIWI pathway genes, which impacts one of their functions: DNA methylation level over LINE-1 promoters is higher in preneoplastic testis tissues adjacent to nonseminomas than those adjacent to seminomas. This finding might imply distinct routes for development of the two types of TGCTs and could be used as a novel diagnostic marker, possibly, noninvasively. Finally, we studied the role of CpG island methylation in expression of PIWI genes in patient samples and using in vitro experiments in cell line models: a more complex interrelation between DNA methylation and expression of the corresponding genes was revealed.

\section{INTRODUCTION}

Testicular germ cell tumors (TGCTs) are heterogeneous cancers classified into less invasive seminomas and more aggressive nonseminomas and are believed to be caused by both genetic and environmental factors [1]. Apart from distinct histological features, seminomas and nonseminomas also differ in their prognosis and choice of treatment strategy [2]. These two types of TGCTs were shown to have numerous epigenetic differences and distinguishing expression of various biomarkers [3, 4].

Essentially, all TGCTs are deemed to arise because of failure to undergo normal spermatogenesis and through an intermediate stage of carcinoma in situ [5-7]. One of the key players in spermatogenesis is PIWI pathway responsible for epigenetic silencing of retrotransposons $[8,9]$. Importantly, PIWI proteins have been also implicated in development of various types of cancers $[10,11]$. Recently, Ferreira et al. [12] demonstrated CpG island (CGI) hypermethylation and a concomitant decrease in expression level of PIWI pathway genes in TGCTs compared to the testes of healthy individuals, suggesting a role of PIWI in TGCT tumorigenesis.

In this work, we extended these data by studying an intermediate preneoplastic stage of TGCT development, which contains carcinoma in situ, i.e. premalignant testis tissues adjacent to TGCTs. We have found distinguishing 
features of spermatogenesis and PIWI machinery function in the two types of TGCTs. Firstly, expression of germ cell marker $D D X 4$ suggests that extent of spermatogenesis in premalignant testis adjacent to nonseminoma is comparable to that in healthy testis. On the other hand, premalignant testis adjacent to seminoma display downregulation of $D D X 4$ expression. Furthermore, expression of PIWIL1/2/4 and DNA methylation level of LINE-1 promoters were found to coincide with this pattern: higher in tissues adjacent to nonseminoma than seminoma. This finding might imply different causes for development of these two types of TGCTs and could be used as a novel diagnostic marker. We additionally studied epigenetic regulation of PIWI machinery genes expression in patient samples and in vitro in cell line models: role for PIWIL1/2 CGI methylation appears to be more complex than it was proposed.

\section{RESULTS AND DISCUSSION}

\section{Expression levels of germ cell marker DDX4 and PIWI genes are different in preneoplastic testis tissues adjacent to seminoma and nonseminoma}

In order to gain more insights into the role of PIWI machinery in TGCT tumorigenesis, apart from just normal and TGCT samples we also used an intermediate preneoplastic stage - testis tissues adjacent to TGCT (CIS samples). This way, we tracked normalto-malignant transition: from $\mathrm{N}$ samples (healthy testis tissues, $n=5$ ) through CIS samples (carcinoma in situ-riddled testis tissues adjacent to TGCT, $n=22$ ) to overt TGCT samples both for seminomas and for nonseminomas (Figure 1A).

Importantly, since each CIS sample was matched to the corresponding TGCT, we were also able to take into account patient-to-patient heterogeneity, which is an emerging feature of tumorigenesis [13-15]. Additionally, we looked into expression of germ cell marker $D D X 4$ (human homologue of Drosophila VASA) to assess for the extent of spermatogenesis and carcinoma in situ markers POU5F1 (OCT3/4) and NANOG to evaluate the degree of testicular dysgenesis in the samples $[16,17]$. This way we could confirm estimates about the histological composition of the samples under study.

Firstly, expression level of carcinoma in situ markers gradually increases from $\mathrm{N}$ through CIS samples to TGCT for both seminomas and nonseminomas (Figure 1B), which reflects accumulation of carcinoma in situ in tissues along the normal-to-malignant transition.

Secondly, although expression of $D D X 4$ is significantly lower in TGCT samples compared to normal testis tissues (Figure 1B) implying deteriorated spermatogenesis, this germ cell marker expression is mostly retained in preneoplastic testis tissues adjacent to nonseminoma unlike those adjacent to seminoma.
Importantly, this pattern appears to be followed by PIWI proteins (Figure 1C). Spearman's correlation coefficient for the expression level of PIWIL1/2/4 and DDX4 genes in normal and premalignant tissues ( $\mathrm{N}$ and NT) is as high as 0.85-0.99 (Supplementary Data File 1), suggesting that expression of PIWI proteins, in fact, reflects the content of germ cells in the samples. It might also imply coordinated regulation of $D D X 4$ and PIWI proteins expression, which is in agreement with the fact that DDX4 has been shown to be involved in piRNA pathway [18].

Thus, by comparing not only healthy testis tissues ( $\mathrm{N}$ samples) and TGCTs but also through studying an intermediate premalignant stage represented by testis tissue adjacent to tumors (CIS samples) we revealed the distinguishing expression patterns for both $D D X 4$ and PIWI proteins. Specifically, these genes are still expressed in CIS samples adjacent to nonseminomas, but are downregulated in those adjacent to seminomas, which could be indicative of different tumorigenesis routes for these two types of TGCTs. Possibly, loss of PIWI machinery function is a prerequisite of germ cell malignization into seminoma, unlike nonseminoma.

One of the prominent features of the obtained data is remarkable patient-to-patient variability, even among normal testes (Ns, Figure $1 \mathrm{~B}$ and $1 \mathrm{C}$ ). This is also evident in the cohort in the study by Ferreira et al. [12]. In order to assess the degree of gene expression heterogeneity among patients, we also performed paired statistical testing (Wilcoxon signed-rank test) of matched CIS/TGCT samples (Figure $1 \mathrm{~B}$ and 1C). The results give more significant evidence (lower $P$ values) to make conclusions on patterns of expression changes. While the expression level of PIWIL1/2/4, DDX4 and carcinoma in situ markers is highly variable among patients, the change patterns in CIS/TGCT matched samples are more consistent across the cohort if regarded in each patient individually. Therefore, the use of matched samples allows minimizing contribution of expression heterogeneity between patients.

We next investigated whether the expression of PIWIL1, PIWIL2 or PIWIL4 in TGCTs correlated with that of $S O X 2$ or $S O X 17$. These two SOX gene family members play important biological roles in germ cell development: $S O X 2$ is involved in maintaining pluripotency $[19,20]$, while $S O X 17$ is a key specification factor for the germ cell fate [21]. Their functions are also relevant to the histological features of TGCTs: more pluripotent embryonal carcinomas (nonseminomas) overexpress $S O X 2$ and more germ cell-like seminomas overexpress $S O X 17$. Consequently, these proteins were previously proposed as markers for the two types of TGCTs [22].

Our analysis showed that the most significant correlation was between PIWIL2 and SOX17 (Supplementary Data File 2). This fact is consistent with a more germ cell-like gene expression pattern in seminomas [23], since PIWIL2 is a component of piRNA pathway, 
whose expression is typically restricted to the germ line. Importantly, PIWIL2 expression was upregulated in seminomas and downregulated in nonseminomas compared to the matched CIS samples (Figure 1C). Accordingly, PIWIL2 transcription level was higher in seminomas than in nonseminomas (Supplementary Data File 3) as in previous reports $[24,25]$. This PIWIL2 expression pattern was also observed in The Cancer Genome Atlas cohort (Supplementary Data File 3). This finding might have some biological relevance, since PIWIL2 was shown to be involved in carcinogenesis as well $[10,11,26]$.

We performed ROC curve analysis in order to assess sensitivity and specificity of PIWIL2 expression level as a biomarker to distinguish between seminomas and nonseminomas. AUC (area under the curve) values for the cohort used in this study (Figure 2A) and for The Cancer Genome Atlas cohort (Figure 2B) indicate that PIWIL2 could be a possible candidate to extend current panels of routinely used TGCT biomarkers [4].

\section{LINE-1 promoter methylation level is affected by PIWI genes expression and could serve as a novel diagnostic marker}

One of the major roles of PIWI proteins in spermatogenesis is maintenance of genomic stability through epigenetic silencing of retrotransposons [27]. It is known that deregulation of retrotransposon expression may lead to neoplastic transformations in various types of cancers [28-30]. Therefore, we obtained integral methylation level of LINE-1 promoters in the samples under study to see whether expression level of PIWI genes impacts their function. Indeed, methylation level of LINE- 1 correlates with expression of PIWI genes: promoters of these retrotransposons are hypermethylated in premalignant testis tissues adjacent to nonseminomas unlike those adjacent to seminomas (Figure 3). Importantly, since DNA methylation is a stable biomarker, it could be used as a novel and robust diagnostic tool for distinguishing between seminomas and nonseminomas, possibly, noninvasively

A

\begin{tabular}{|c|c|c|c|c|}
\hline Seminoma $\leftarrow$ & $\begin{array}{l}\text { Premalignant testis } \\
\text { tissue adjacent to seminoma }\end{array}$ & $\begin{array}{l}\text { Healthy testis } \\
\text { tissue }\end{array}$ & $\rightarrow \underset{\text { tissue adjacent to nonseminoma }}{\text { Premalignant testis }} \rightarrow \overrightarrow{ }$ & Nonseminoma \\
\hline TGCT (SE) & CIS (SE) & $\mathrm{N}$ & CIS (NS) & TGCT (NS) \\
\hline
\end{tabular}

B

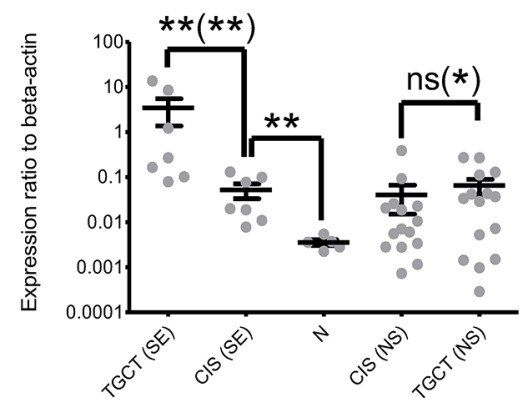

C

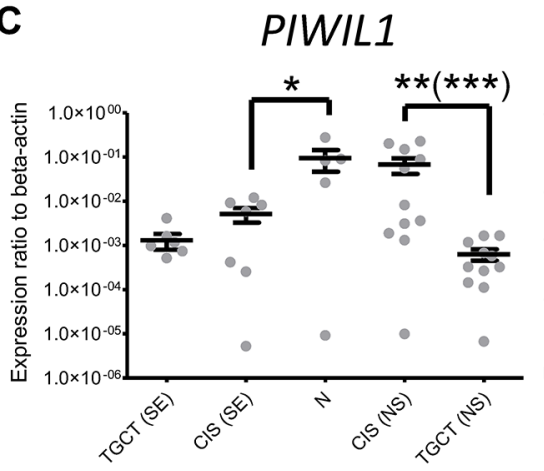

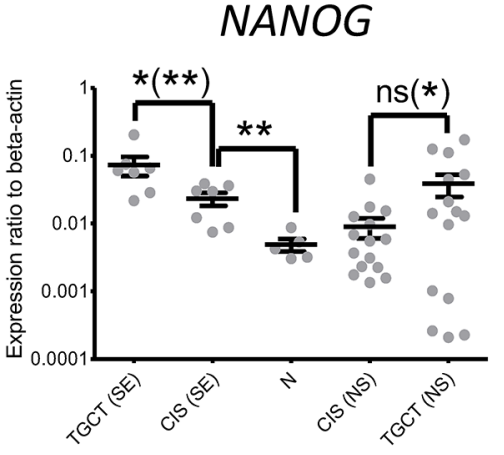

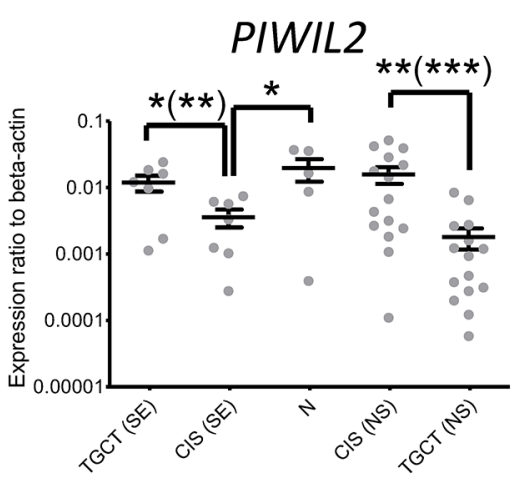

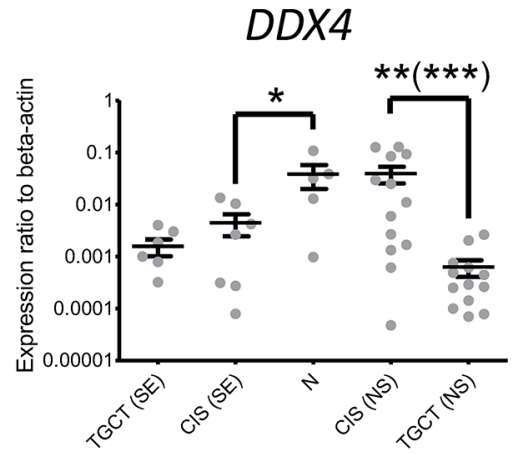

PIWIL4

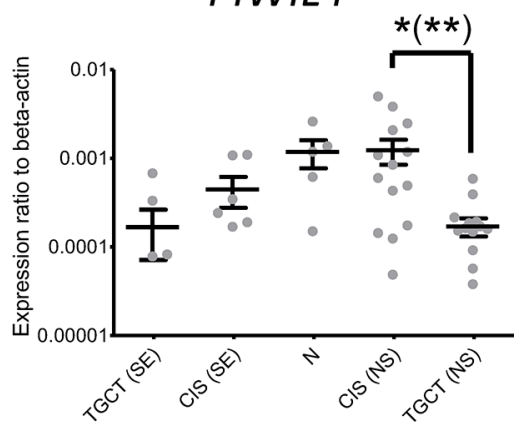

Figure 1: Expression of CIS and germ cell markers and PIWI protein genes in testis and testicular germ cell tumors. (A) Schematic description of the sample types used in the work and the approach to study normal-to-malignant transition. (B) Expression of carcinoma in situ markers POU5F1 (OCT3/4) and NANOG and germ cell marker DDX4. (C) Expression of PIWI protein genes PIWIL1, PIWIL2, and PIWIL4. Average value and SEM (Standard Error of the Mean) are shown for all samples. $P$ value summary for Mann-Whitney non-paired $U$ test and paired Wilcoxon signed-rank test (in brackets) are shown only for significant differences. Due to logariphmic scale several near-zero values are not depicted: DDX4 - 1 TGCT(SE), PIWIL1 - 1 TGCT(SE), PIWIL4 - 3 TGCT(SE), 1 CIS (SE), 1 CIS (NS) and 2 TGCT(NS). 
(e.g., in seminal fluid). Importantly, this finding could also imply the fact that loss of LINE-1 silencing is a contributor into seminoma but not nonseminoma development.

\section{More complex role of DNA methylation in regulation of PIWIL1 and PIWIL2 expression}

Ferreira et al. [12] also examined CGI methylation of PIWI genes promoters and reported an association between CGI methylation and transcriptional repression of the corresponding genes in TGCT samples. Since we could expand these data by examining CIS samples (preneoplastic testis tissue adjacent to tumors), we assessed PIWIL1 and PIWIL2 CGI methylation status in our cohort. The results obtained for healthy testis tissues (N samples) and TGCTs were in line with those by Ferreira et al. (Figure 4A and 4B) [12]. However, premalignant testis samples adjacent to both seminoma and nonseminoma were highly methylated (Figure 4A and 4B), despite the fact that PIWIL1 and PIWIL2 are expressed in CIS samples adjacent to nonseminomas at the

\section{A}
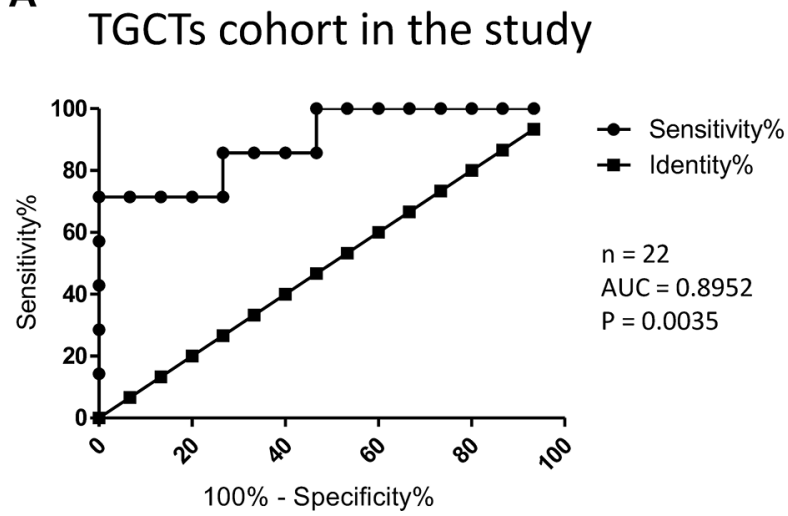

level comparable with healthy testis tissues ( $\mathrm{N}$ samples). This disagreement could be caused by a more complex link between PIWIL1 and PIWIL2 CGI methylation and gene expression.

To test for this hypothesis, we performed two experiments in vitro in cancer cell lines. In the first assay, lung carcinoma A549 cells (TGCT-irrelevant control) and embryonal carcinoma TERA1 cells (nonseminomalike cells) were transiently transfected with a luciferase reporter construct containing unmethylated or methylated PIWIL1/2 promoters designed according to Dell et al. [31]. Importantly, these constructs were exogenous for cells and devoid of chromatin "memory". As expected, the methylated promoters exerted no activity; however, the unmethylated PIWIL1 promoter was only active in A549 cells and unmethylated PIWIL2 promoter was only active in TERA1 cells (Figures 5A and 4B), suggesting that CGI unmethylated status is necessary but not sufficient for PIWIL1/2 promoter activity, possibly, because of lack of transcriptional factors essential for transcriptional activation.
B

\section{The Cancer Genome Atlas cohort}

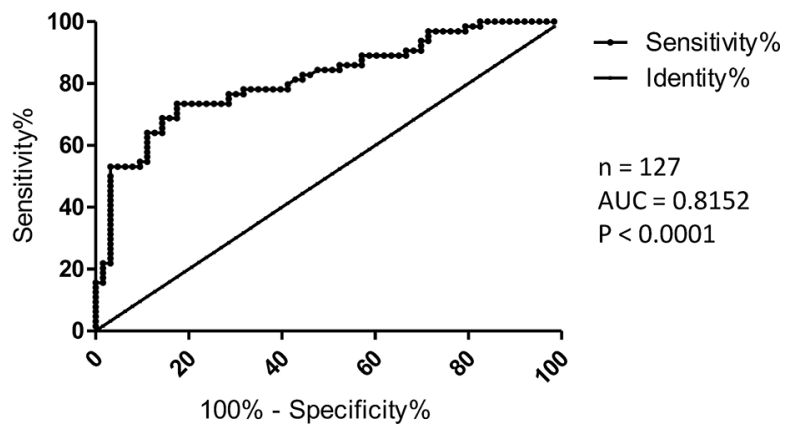

Figure 2: Assessment of sensitivity and specificity of PIWIL2 expression level as a biomarker to distinguish between seminomas and nonseminomas. ROC curve analysis was performed and AUC (Area Under the Curve) was calculated for the cohorts from the present study (A) and The Cancer Genome Atlas project (B). The number of samples in each cohort and $P$ values are indicated.

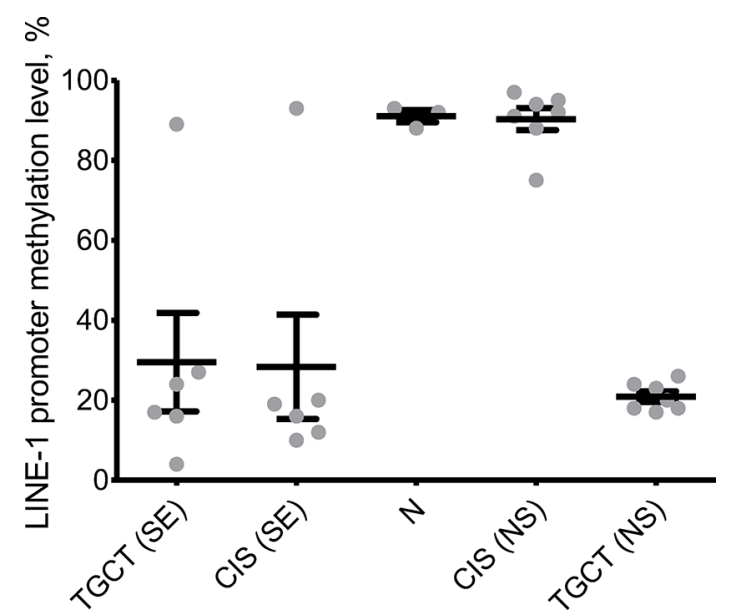

Figure 3: Integral methylation status of LINE-1 CpG islands. Melting curve analysis was used to obtain methylation level in healthy testis tissues (N), premalignant testis tissues (CIS samples) and testicular germ cell tumors (TGCT). SE - seminoma, NS - nonseminoma. 
In the second experiment, we wanted to assess how promoter DNA methylation influences expression of PIWIL1 and PIWIL2 in their natural genomic DNA context. While studying PIWIL1 and PIWIL2 promoters in the genome of TERA1 and A549 cell lines, we found them to be highly methylated. In order to achieve their demethylation, these cell lines were treated with inhibitors of DNA methyltransferase (5-Aza-2'-deoxycytidine) and histone deacetylases (trichostatin A) [32]. The combined drug treatment protocol was used as the most efficient method to ensure almost complete loss of methylated CpGs from around methylated promoters of PIWIL1/2 (Figure 6A).

As expected, in A549 cells promoter demethylation led to an increase in $\mathrm{H} 3 \mathrm{~K} 4 \mathrm{me} 3$ (histone methylation mark of active transcription, Figure $6 \mathrm{~B}$ ) and upregulated PIWIL1 and PIWIL2 expression (Figure 6C).

However, in TERA1 cells PIWIL2 transcription was already active before drug treatment and in presence of its highly methylated promoter (Figure 6A and 6C). Druginduced demethylation did not produce any changes in transcription for both PIWIL1 and PIWIL2 (Figure 6C). That is a surprising result because $\mathrm{H} 3 \mathrm{~K} 4 \mathrm{me} 3$ chromatin marks for active transcription were gained on both promoters (Figure 6B). Among plausible explanations for the results in TERA1 cell line may be presence of other than $\mathrm{CpG}$ methylation mechanisms that maintain activating or repressive chromatin environment on PIWIL1/2 promoters.

Overall, these seemingly conflicting findings in in vitro experiments imply a more complex link between CGI methylation in the promoter regions and PIWIL1/2 transcription and, possibly, interplay with other expression regulatory systems. That is not an entirely new observation, since "atypical" promoters (active, while being heavily methylated) have been described in the course of spermatogenesis [33]. Moreover, active transcription from promoters associated with methylated CGIs was also shown in other contexts [34-38].

\section{CONCLUSION}

In this work, we extended results from previous studies on PIWI machinery proteins expression in TGCTs by introducing an intermediate premalignant stage. Namely, we found that PIWIL1/2/4 and DDX4 are concertedly expressed in preneoplastic testis tissue adjacent to nonseminoma but are downregulated in those adjacent to seminoma. Possibly, the loss of spermatogenesis and/or PIWI machinery might be required for seminoma development. This attrition could also take place at an earlier stage of neoplastic transformation into seminoma, unlike nonseminoma. Importantly, whether it is a cause or a consequence remains to be answered in further studies. In addition, TGCTs accompanied by cryptorchidism without orchiopexy are most commonly seminomas [39-41]. Therefore, reasons for impaired spermatogenesis in uncorrected cryptorchidism and development of seminoma might be similar and connected with piRNA pathway function.

Furthermore, PIWI proteins expression pattern in premalignant testis was found to have an impact on piRNA machinery function - methylation level of LINE- 1 promoters, which could directly impact the genomic stability and, in turn, promote malignization of germ cells. Apart from that, this observation can be used as a novel diagnostic biomarker for the two types of TGCTs.

Moreover, use of matched CIS/TGCT pairs also allowed minimizing data variability and obtaining a refined picture devoid of patient-to-patient heterogeneity. Finally, a more complex role of PIWIL1/2 CGI methylation in expression of corresponding genes was revealed.
A

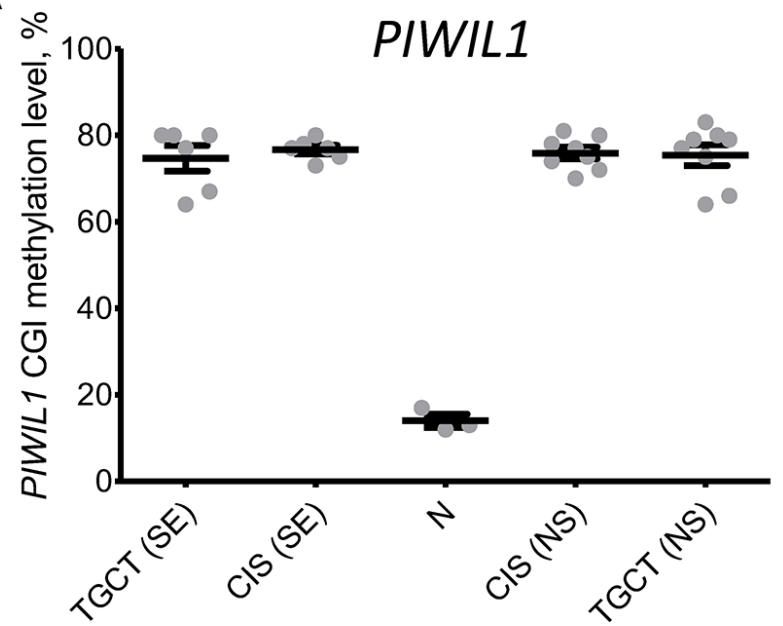

B

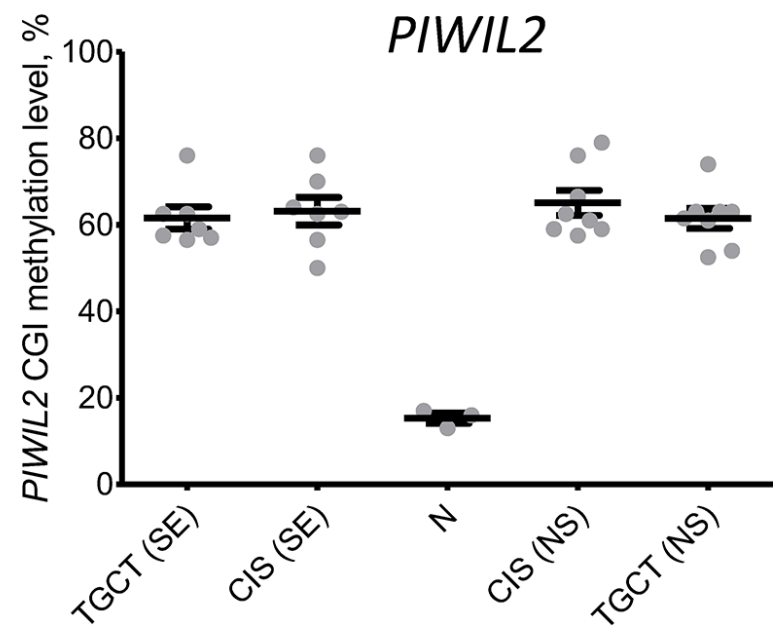

Figure 4: Integral methylation status of PIWIL1 and PIWIL2 CpG islands. Melting curve analysis was used to obtain methylation level of PIWIL1 (A) and PIWIL2 (B) CpG islands in healthy testis tissues (N samples), premalignant testis tissues (CIS samples) and testicular germ cell tumors (TGCT). SE - seminoma, NS - nonseminoma. 
A

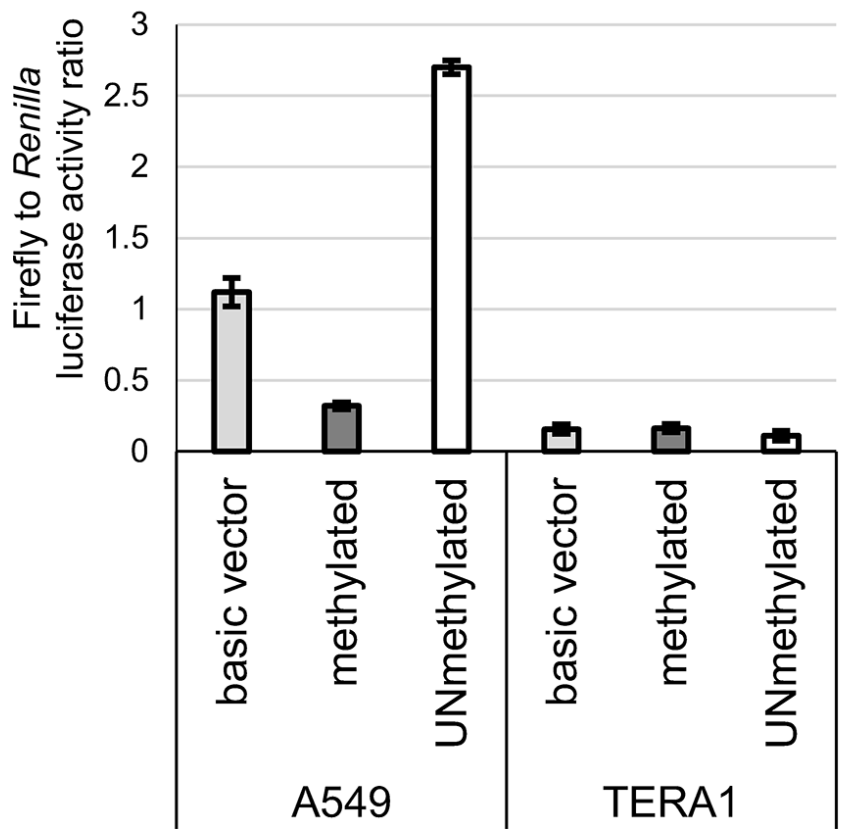

B

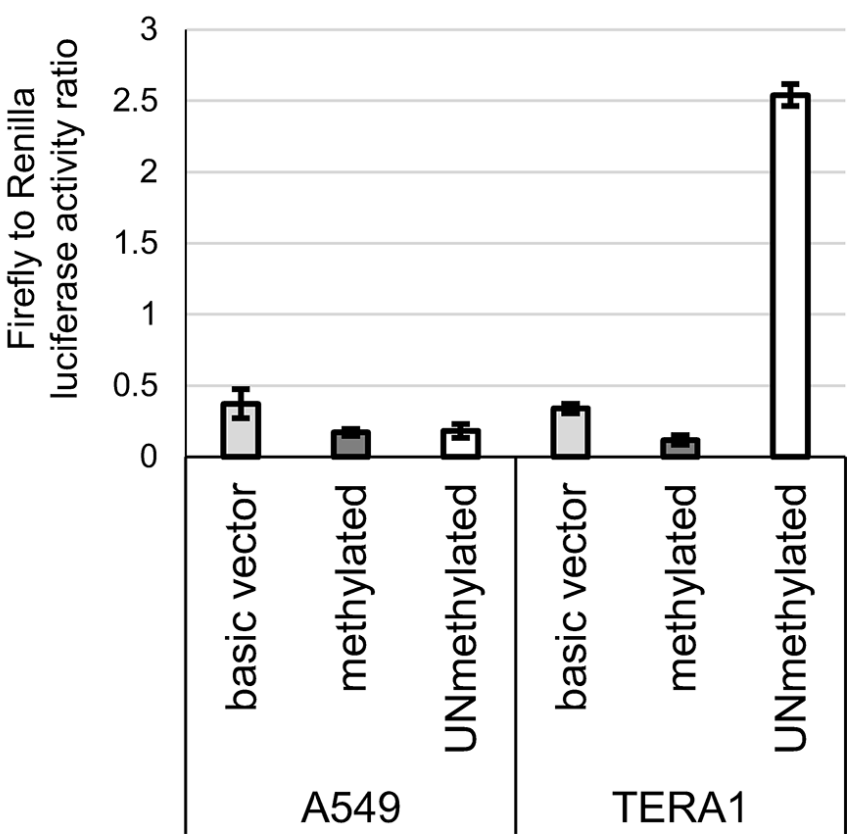

Figure 5: Luciferase reporter assay with methylated or unmethylated PIWIL1 and PIWIL2 promoters. Luciferase reporter constructs pGL4.10 with either methylated or unmethylated PIWIL1 (A) or PIWIL2 (B) promoter were transfected into TERA1 and A549 cells. The basic vector (no promoter control) was used as the reference.

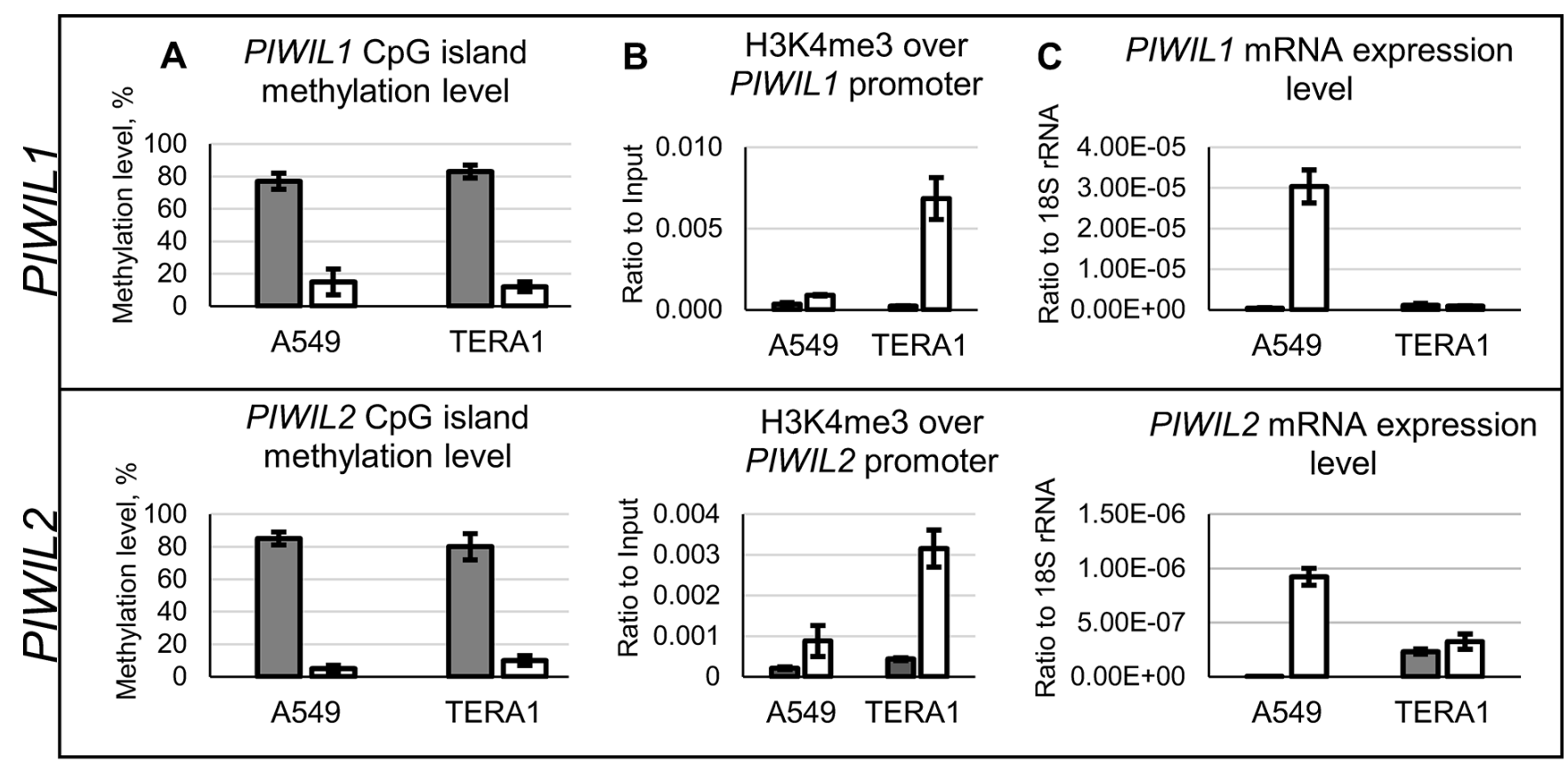

$\square$ No treatment $\square$ 5-aza-dC + TSA

Figure 6: Drug-induced demethylation of genomic promoters of PIWIL1 and PIWIL2. TERA1 and A549 cells were treated with 5-Aza-2'-deoxycytidine (5-aza-dC) and trichostatin A (TSA) and analyzed for promoter CpG methylation (A), chromatin modifications of active transcription start sites (H3K4me3, trimethylated lysine 4 in histone 3 ) along promoters (B), and transcription level (C) of PIWIL1 and PIWIL2 genes. 


\section{MATERIALS AND METHODS}

\section{Sample collection}

Twenty two pairs of TGCT tissues and corresponding adjacent normal testicular parenchyma were obtained from orchiectomy specimens: 7 seminomas (patient age ranges between 19 y.o. and 51 y.o. with a median of 34 y.o.) and 15 nonseminomas (patient age ranges between 18 y.o. and 49 y.o. with a median of 28 y.o.). The 15 nonseminomas included 4 teratomas, 1 choriocarcinoma, 1 embryonal carcinoma and 9 TGCTs with mixed histology. 5 samples of normal testis tissue were obtained from prostate cancer patients undergoing surgical castration (patient age ranges between 53 y.o. and 84 y.o. with a median of 68 y.o.). The samples were immediately frozen in liquid nitrogen. All patients provided written informed consent according to the federal law, and the study was approved by the ethical committees of the Shemyakin-Ovchinnikov Institute of Bioorganic Chemistry of the Russian Academy of Sciences and Blokhin Russian Cancer Research Center after reviewing patients' consent and information forms.

\section{Gene expression analysis by qRT-PCR}

Total RNA extraction and purification was performed with Trizol reagent (Invitrogen, USA). cDNA synthesis was performed with MintReverse Transcriptase and following qPCRs with qPCRmix-HS SYBR system (Evrogen, Russia) on Lightcycler 480 (Roche, USA) according to the manufacturers' instructions. Primer pairs used in amplification are listed in Supplementary Data File 4 . The assays were performed in triplicates.

\section{DNA methylation analysis by melting curve assay}

Melting curve analysis was used to assess the integral level of DNA methylation for LINE-1 and PIWIL1/2 promoter CGIs as previously described [42]. Primers are listed in Supplementary Data File 5 and calibration curves are shown in Supplementary Data File 6. Biological duplicates (cell line experiments only) and technical triplicates were used to ensure reproducibility.

\section{Luciferase reporter assay}

PIWIL1/2 promoter regions were cloned (primers are listed in Supplementary Data File 7) and ligated upstream of the luciferase reporter gene in pGL4.10 (Promega, USA) in either methylated or unmethylated state (according to Dell et al. [31]). Transient cotransfection with pRL-TK reference vector (Promega, USA) coding for Renilla reniformis luciferase was performed using Lipofectamin 2000 (Invitrogen, USA) according to the manufacturers' protocols. Relative luciferase activity was obtained. Biological and technical duplicates were used to ensure reproducibility.

\section{Cell lines and treatment with 5-Aza-dC and trichostatin A}

Cell lines TERA1 (ATCC HTB-105) and A549 (ATCC CCL-185) were purchased from ATTC (USA) and maintained in DMEM/F12 (1:1) (Invitrogen, USA) supplemented with 10\% FCS (Invitrogen). For DNA demethylation experiments, cells were grown for 4 days in the presence of freshly prepared $5 \mu \mathrm{M} 5$-Aza-2'deoxycytidine (Sigma, USA); the medium containing 5-Aza$\mathrm{dC}$ was changed daily. Trichostatin A (Sigma, USA) was added at the concentration of $200 \mathrm{nM} 8 \mathrm{~h}$ before analysis. Untreated cell cultures were used as controls. Biological and technical triplicates were used to ensure reproducibility.

\section{Chromatin immunoprecipitation (ChIP) assay}

ChIP was performed as described earlier [43] using antibodies to trimethylated lysine 4 in human histone 3 (H3K4me3; ab8580, Abcam, USA). Biological and technical triplicates were used to ensure reproducibility. Primer pairs are listed in Supplementary Data File 8.

\section{ACKNOWLEDGMENTS}

We thank Dr. Lev G. Nikolaev and Prof. Eugene D. Sverdlov at the Shemyakin-Ovchinnikov Institute of Bioorganic Chemistry for critical reading of the manuscript, Dr. Sergey B. Akopov for assistance in cell culture work, and Olesya V. Maslova for assistance in sample collection.

\section{Abbreviations}

TGCT, testicular germ cell tumor; ChIP, chromatin immunoprecipitation; H3K4me3, histone 3 lysine 4 trimethylation.

\section{Authors' contributions}

IG and TA conceived the study and wrote the manuscript. IG, SK, YS, MZ, and ES conducted the experiments. AK and AT performed surgical sample collection and histological evaluation. All authors have read and approved the final version of the manuscript.

\section{GRANT SUPPORT}

This study was supported by the Russian Foundation for Basic Research (no. 14-04-32314-mol a) and the program of the Presidium of the Russian Academy of Sciences "Molecular and Cellular Biology." 


\section{CONFLICTS OF INTEREST}

The authors declare no competing interests.

\section{REFERENCES}

1. Looijenga LH, Van Agthoven T, Biermann K. Development of malignant germ cells - the genvironmental hypothesis. The International journal of developmental biology. 2013; 57:241-253.

2. Stevenson SM, Lowrance WT. Epidemiology and Diagnosis of Testis Cancer. The Urologic clinics of North America. 2015; 42:269-275.

3. Kristensen DG, Skakkebaek NE, Rajpert-De Meyts E, Almstrup K. Epigenetic features of testicular germ cell tumours in relation to epigenetic characteristics of foetal germ cells. The International journal of developmental biology. 2013; 57:309-317.

4. Chieffi P. Recent advances in molecular and cell biology of testicular germ-cell tumors. International review of cell and molecular biology. 2014; 312:79-100.

5. Rijlaarsdam MA, Looijenga LH. An oncofetal and developmental perspective on testicular germ cell cancer. Seminars in cancer biology. 2014; 29C:59-74.

6. Rajpert-De Meyts E, Nielsen JE, Skakkebaek NE, Almstrup K. Diagnostic markers for germ cell neoplasms: from placental-like alkaline phosphatase to micro-RNAs. Folia Histochem Cytobiol. 2015.

7. Skakkebaek NE. Possible carcinoma-in-situ of the testis. Lancet. 1972; 2:516-517.

8. Aravin AA, Sachidanandam R, Girard A, Fejes-Toth K, Hannon GJ. Developmentally regulated piRNA clusters implicate MILI in transposon control. Science. 2007; 316:744-747.

9. Iwasaki YW, Siomi MC, Siomi H. PIWI-Interacting RNA: Its Biogenesis and Functions. Annu Rev Biochem. 2015; 84:405-433.

10. Suzuki R, Honda S, Kirino Y. PIWI Expression and Function in Cancer. Frontiers in genetics. 2012; 3:204.

11. Ross RJ, Weiner MM, Lin H. PIWI proteins and PIWIinteracting RNAs in the soma. Nature. 2014; 505:353-359.

12. Ferreira HJ, Heyn H, Garcia del Muro X, Vidal A, Larriba S, Munoz C, Villanueva A, Esteller M. Epigenetic loss of the PIWI/piRNA machinery in human testicular tumorigenesis. Epigenetics : official journal of the DNA Methylation Society. 2014; 9:113-118.

13. Brait M, Maldonado L, Begum S, Loyo M, Wehle D, Tavora FF, Looijenga LH, Kowalski J, Zhang Z, Rosenbaum E, Halachmi S, Netto GJ, Hoque MO. DNA methylation profiles delineate epigenetic heterogeneity in seminoma and nonseminoma. British journal of cancer. 2012; 106:414-423.

14. Atlasi Y, Looijenga L, Fodde R. Cancer stem cells, pluripotency, and cellular heterogeneity: a WNTer perspective. Current topics in developmental biology. 2014; 107:373-404.
15. Magee JA, Piskounova E, Morrison SJ. Cancer stem cells: impact, heterogeneity, and uncertainty. Cancer cell. 2012; 21:283-296.

16. Looijenga LH, Stoop H, de Leeuw HP, de Gouveia Brazao CA, Gillis AJ, van Roozendaal KE, van Zoelen EJ, Weber RF, Wolffenbuttel KP, van Dekken H, Honecker F, Bokemeyer C, et al. POU5F1 (OCT3/4) identifies cells with pluripotent potential in human germ cell tumors. Cancer research. 2003; 63:2244-2250.

17. Hoei-Hansen CE, Almstrup K, Nielsen JE, Brask Sonne S, Graem N, Skakkebaek NE, Leffers H, RajpertDe Meyts E. Stem cell pluripotency factor NANOG is expressed in human fetal gonocytes, testicular carcinoma in situ and germ cell tumours. Histopathology. 2005; 47:48-56.

18. Chuma S, Nakano T. piRNA and spermatogenesis in mice. Philos Trans R Soc Lond B Biol Sci. 2013; 368:20110338.

19. Wang J, Rao S, Chu J, Shen X, Levasseur DN, Theunissen TW, Orkin SH. A protein interaction network for pluripotency of embryonic stem cells. Nature. 2006; 444:364-368.

20. Pan G, Li J, Zhou Y, Zheng H, Pei D. A negative feedback loop of transcription factors that controls stem cell pluripotency and self-renewal. FASEB J. 2006; 20:1730-1732.

21. Irie N, Weinberger L, Tang WW, Kobayashi T, Viukov S, Manor YS, Dietmann S, Hanna JH, Surani MA. SOX17 Is a Critical Specifier of Human Primordial Germ Cell Fate. Cell. 2015; 160:253-268.

22. de Jong J, Stoop H, Gillis AJ, van Gurp RJ, van de Geijn GJ, Boer M, Hersmus R, Saunders PT, Anderson RA, Oosterhuis JW, Looijenga LH. Differential expression of SOX17 and SOX2 in germ cells and stem cells has biological and clinical implications. The Journal of pathology. 2008; 215:21-30.

23. Alagaratnam S, Lind GE, Kraggerud SM, Lothe RA, Skotheim RI. The testicular germ cell tumour transcriptome. International journal of andrology. 2011; 34:e133-150; discussion e150-131.

24. Lee JH, Schutte D, Wulf G, Fuzesi L, Radzun HJ, Schweyer S, Engel W, Nayernia K. Stem-cell protein Piwil2 is widely expressed in tumors and inhibits apoptosis through activation of Stat3/Bcl-XL pathway. Human molecular genetics. 2006; 15:201-211.

25. Gainetdinov IV, Skvortsova YV, Stukacheva EA, Bychenko OS, Kondratieva SA, Zinovieva MV, Azhikina TL. Expression profiles of PIWIL2 short isoforms differ in testicular germ cell tumors of various differentiation subtypes. PloS one. 2014; 9:e112528.

26. Tan Y, Liu L, Liao M, Zhang C, Hu S, Zou M, Gu M, Li X. Emerging roles for PIWI proteins in cancer. Acta Biochim Biophys Sin (Shanghai). 2015; 47:315-324.

27. Siomi MC, Sato K, Pezic D, Aravin AA. PIWI-interacting small RNAs: the vanguard of genome defence. Nature reviews Molecular cell biology. 2011; 12:246-258. 
28. Sciamanna I, Gualtieri A, Piazza PF, Spadafora C. Regulatory roles of LINE-1-encoded reverse transcriptase in cancer onset and progression. Oncotarget. 2014; 5:8039-8051. doi: 10.18632/oncotarget.2504.

29. Carreira PE, Richardson SR, Faulkner GJ. L1 retrotransposons, cancer stem cells and oncogenesis. FEBS J. 2014; 281:63-73.

30. Hancks DC, Kazazian HH, Jr. Active human retrotransposons: variation and disease. Current opinion in genetics \& development. 2012; 22:191-203.

31. Dell G, Charalambous M, Ward A. In vitro methylation of specific regions in recombinant DNA constructs by excision and religation. Methods in molecular biology. 2001; 181:251-258.

32. Cameron EE, Bachman KE, Myohanen S, Herman JG, Baylin SB. Synergy of demethylation and histone deacetylase inhibition in the re-expression of genes silenced in cancer. Nature genetics. 1999; 21:103-107.

33. Hammoud SS, Low DH, Yi C, Carrell DT, Guccione E, Cairns BR. Chromatin and transcription transitions of mammalian adult germline stem cells and spermatogenesis. Cell stem cell. 2014; 15:239-253.

34. Raynal NJ, Si J, Taby RF, Gharibyan V, Ahmed S, Jelinek J, Estecio MR, Issa JP. DNA methylation does not stably lock gene expression but instead serves as a molecular mark for gene silencing memory. Cancer research. 2012; 72:1170-1181.

35. Metivier R, Gallais $\mathrm{R}$, Tiffoche $\mathrm{C}$, Le Peron $\mathrm{C}$, Jurkowska RZ, Carmouche RP, Ibberson D, Barath P, Demay F, Reid G, Benes V, Jeltsch A, Gannon F, et al. Cyclical DNA methylation of a transcriptionally active promoter. Nature. 2008; 452:45-50.
36. Weber M, Hellmann I, Stadler MB, Ramos L, Paabo S, Rebhan M, Schubeler D. Distribution, silencing potential and evolutionary impact of promoter DNA methylation in the human genome. Nature genetics. 2007; 39:457-466.

37. Meissner A, Mikkelsen TS, Gu H, Wernig M, Hanna J, Sivachenko A, Zhang X, Bernstein BE, Nusbaum C, Jaffe DB, Gnirke A, Jaenisch R, Lander ES. Genome-scale DNA methylation maps of pluripotent and differentiated cells. Nature. 2008; 454:766-770.

38. Jeltsch A, Jurkowska RZ. New concepts in DNA methylation. Trends in biochemical sciences. 2014; 39:310-318.

39. Batata MA, Whitmore WF, Jr., Chu FC, Hilaris BS, Loh J, Grabstald H, Golbey R. Cryptorchidism and testicular cancer. The Journal of urology. 1980; 124:382-387.

40. Wood HM, Elder JS. Cryptorchidism and testicular cancer: separating fact from fiction. The Journal of urology. 2009; $181: 452-461$.

41. Agoulnik AI, Huang Z, Ferguson L. Spermatogenesis in cryptorchidism. Methods in molecular biology. 2012; 825:127-147.

42. Smith E, Jones ME, Drew PA. Quantitation of DNA methylation by melt curve analysis. BMC cancer. 2009; 9:123.

43. Orlando V. Mapping chromosomal proteins in vivo by formaldehyde-crosslinked-chromatin immunoprecipitation. Trends in biochemical sciences. 2000; 25:99-104. 